\title{
Obtención de modelos 3D de estructuras de patrimonio cultural-urbano con el uso de tecnología escáner láser y SIG, como instrumentos para el desarrollo local
}

Obtaining 3D models of cultural-urban heritage structures with the use of laser scanner technology and GIS, as instruments for local development

Luis Alejandro Velastegui Cáceres. ${ }^{1}$, Julia Desiree Velastegui Cáceres. ${ }^{2}$, Vilma Fernanda Noboa Silva. ${ }^{3} \&$ Manuel Fabian Moyon Gusqui. ${ }^{4}$

Recibido: 02-09-2019 / Revisado: 05-10-209 /Aceptado: 24-10-2019/ Publicado: 04-11-2019

\begin{abstract}
.
DOI: https://doi.org/10.33262/cienciadigital.v3i4.1.980

The present project offers the use of laser technology to obtain three-dimensional models, with high precision in a short period of time. In Ecuador there are few projects where this technology is used to obtain geospatial data. This project shows the utility of the laser scanner system using the Faro Scanner Focus3D 130 for the registration of Cultural Heritage monuments. The advantages of using laser scanner technology is the feasibility for taking geospatial data with a high level of detail where the error range is $2 \mathrm{~mm}$. This allowed the development of virtual environments of the facade of "La Casona San Juanista" and the facade of the "La Compañía de Jesús" Church. These Cultural Heritage monuments were selected because of the complexity of their structure, their details and their significance of heritage importance for Ecuador. With
\end{abstract}

\footnotetext{
${ }^{1}$ Universidad Nacional de Chimborazo, Facultad de Ingeniería, Riobamba, Ecuador, lavelastegui@unach.edu.ec

${ }^{2}$ Escuela Superior Politécnica de Chimborazo, Facultad de Recursos Naturales, Riobamba, Ecuador, julia.velastegui@espoch.edu.ec

3 Escuela Superior Politécnica de Chimborazo, Facultad de Recursos Naturales, Riobamba, Ecuador, vilma.noboa@espoch.edu.ec

4 Universidad de las Fuerzas Armadas ESPE, Departamento de Ciencias de la Tierra y la Construcción, Sangolqui, Ecuador, mfmoyon@espe.edu.ec
} 
the obtaining of the 3D models of these patrimonial monuments demonstrated that with this type of technology it is possible to generate three-dimensional models in the Cultural Heritage area in a short time and at the same time with a high level of detail and millimeter precision.

Keywords: GIS; Cultural Heritage; Local development; 3D models

\section{Resumen.}

En el presente proyecto se propone la utilización de la tecnología láser para obtener modelos tridimensionales, con una alta precisión en un período relativamente corto de tiempo. En Ecuador existen pocos proyectos donde se utiliza esta tecnología para la obtención de datos geoespaciales. La importancia del presente proyecto es mostrar la utilidad del sistema escáner láser utilizando el Faro Láser Scanner Focus3D 130 para el registro de obras de alto valor Patrimonial. Las ventajas al utilizar la tecnología del escáner láser es la factibilidad para la toma de datos geoespaciales con alto nivel de detalle donde el rango de error es de $2 \mathrm{~mm}$. Con ello fue posible el desarrollo de ambientes virtuales de la fachada de "La Casona San Juanista" y la fachada de la Iglesia "La Compañía de Jesús". Estas obras de Patrimonio Cultural fueron seleccionadas por la complejidad de su estructura, sus detalles y por su significativa importancia patrimonial para Ecuador. Con la obtención de los modelos 3D de ambas obras patrimoniales se demuestra que con este tipo de tecnología es posible la generación de modelos tridimensionales en el área de Patrimonio Cultural en corto tiempo y a su vez con alto nivel de detalle y de precisión milimétrica.

Palabras claves: SIG, Patrimonio Cultural, Desarrollo Local, modelos 3D

\section{Introducción.}

El registro geométrico de infraestructuras industriales, civiles y arquitectónicas requiere de herramientas tecnológicas, mismas que se han convertido en apoyo indispensable para la elaboración de planos con un nivel de precisión aceptable. En el manejo de datos geoespaciales se ha trabajado con sistemas $\mathrm{CAD}$, siendo estos útiles para tratar de simular ambientes virtuales principalmente en el ámbito de la construcción; posteriormente aparecen otras plataformas dentro de los Sistemas de Información Geográfica (Apollonio et al. 2012; Piedecausa et al. 2015; Sibato, 2018) los cuales se convierten en herramientas esenciales para modelamientos en tres dimensiones (3D). Sin embargo, esta tecnología no ha sido la más eficiente para obtener una adecuada simulación de la realidad en diferentes áreas (Patrimonio Cultural y Natural); debido a su poca precisión, bajo nivel de detalle y de resolución (Guidi et al 2015, Manaña et al., 2008). Actualmente la tecnología de punta en modelamientos 3D foto-realísticos son los sistemas escáner láser terrestre que surgieron en Europa, Asia, tomando un repunte en Estados Unidos para la fiscalización, supervisión, remodelación y control del deterioro de obras patrimoniales y civiles (Xu et al. 2014). En latinoamérica no 
se contaba con las herramientas tecnológicas adecuadas para disminuir las falencias al momento de realizar un levantamiento de construcciones con precisión y alto nivel de detalle (Martínez et al. 2018).

Los sistemas escáner láser terrestre facilitan la documentación de objetos, edificios y lugares en su forma íntegra y específica con un alto nivel de detalle y con una precisión al milímetro (Echeverría et al. 2017; Santana et al., 2008), permitiendo una comparación adecuada de estas estructuras existentes con los planos de construcción; lo que simplifica de gran manera la planificación en el proceso de construcción o de evaluación de patrimonio cultural en el caso de construcciones históricas o de valor arquitectónico (Bermúdez et al. 2016; Bernat et al. 2014). La documentación de elementos arquitectónicos involucra procesos no únicamente de toma de datos in situ sino también el procesamiento de los datos geoespaciales, presentación, almacenamiento y visualización de la información. La mayoría de las representaciones planimétricas se encuentran en 2D; sin embargo, en la actualidad las representaciones geométricas han evolucionado hacia un registro $3 \mathrm{D}$ mismo que permite obtener un modelo tridimensional que contiene la representación gráfica de la geometría de cada plano de obras civiles y/o arquitectónicas (Manso, 2017).

De hecho, el Distrito Metropolitano de Quito cuenta con uno de los más sobresalientes y mejor conservado Centro Histórico, pero de este Patrimonio Cultural no se tiene registros fidedignos; lo cual es esencial para su manejo y posterior restauración. La limitación de registros geométricos dificulta los procesos de restauración e incluso de turismo. Cabe mencionar que en la actualidad los registros tridimensionales son utilizados para promover el turismo a través de recorridos virtuales. Esta tecnología revoluciona el campo del Patrimonio Cultural; debido a que permite la documentación íntegra y detallada de obras históricas y monumentos, posibilitando la captura masiva de información sobre estos objetos (Redondo y Adas, 2014; Santana et al., 2008). Además, se puede obtener un íntegro control del deterioro de los distintos espacios físicos construidos (Owda et al. 2018; Tamayo y Leite, 2015).

El modelamiento digital en 3D constituye una de las herramientas más efectivas para la documentación, investigación, divulgación y demostración del valor de Patrimonio Cultural de los monumentos (Echeverría et al. 2017). Contar con un registro digital 3D de monumentos patrimoniales contribuye al proceso de restauración cuando las estructuras presentan deterioro por eventos naturales; así también, para remodelar los desperfectos causados por el paso del tiempo. Los modelos 3D permiten promover el turismo y dar a conocer la riqueza patrimonial (Bermúdez et al. 2016; Guidi et al. 2015; Martínez et al. 2018; Maravelakis et al. 2013; Owda et al. 2018). Una de las ventajas de la tecnología escáner láser terrestre es el registro de edificaciones arquitectónicas de alto valor patrimonial sin que exista un impacto físico a estas edificaciones lo que evita cualquier tipo de riesgo de deterioro con este tipo de láser (Manso, 2017). Así también la generación de ambientes virtuales que permiten la interacción y visualización en tiempo real de objetos creados en un ordenador. 
Sin embargo, no sólo deben considerarse como partes primordiales de estos ambientes a los elementos propios de su interfaz (texto, gráficos, sonidos, animaciones, hipervínculos, entre otros), el objetivo fundamental radica en el alcance final obtenido al instaurarlo (Andrade y Narea, 2011; Maravelakis et al. 2013). Las características más importantes que se consideran son la expresión en un lenguaje gráfico tridimensional, el comportamiento dinámico y operación en tiempo real, el funcionamiento basado en la incorporación del usuario en el medio digital, la capacidad de reaccionar ante el usuario ofreciendo en su modo más complejo una experiencia inmersiva, interactiva y multisensorial.

En el contexto urbano las tecnologías geoespaciales tales como Sistemas de Información Geográfica y tecnología láser terrestre (TLS) se aplican para el desarrollo de sistemas de soporte para la toma de decisiones. Riobamba cuenta con obras de alto valor patrimonial, entre ellas La Casona San Juanista caracterizada por sus molduras de inicio del siglo XX, misma que fue restaura para que actualmente se convierta en parte del Campus Centro de la Universidad Nacional de Chimborazo. Su fachada tiene molduras complejas que para registrarlas implica el uso de tecnologías vanguardistas, entre ellas tecnologías geoespaciales, para obtener registros con alta y nivel de detalle.

Este proyecto permite una precisa y completa representación, además del almacenamiento de la realidad de un lugar en las áreas de la construcción histórica representativos sitios para el patrimonio cultural, a través de los modelos tridimensionales obtenidos. Por lo tanto, esta tecnología innova la realización de modelos en corto tiempo y con alta precisión, entregando soluciones en estas tres áreas de estudio y en un futuro poder inventariar el patrimonio cultural como herramienta para el desarrollo local, considerando que alrededor del patrimonio cultural se teje y se desarrolla el patrimonio intangible, el cual se expresa mediante la cultura y tradición de los habitantes cercanos, por lo que son necesarios mecanismos que permitan visualizar, disponer y popularizar esta información del ambiente urbano histórico-cultural, donde se tendrá una mejor lectura del territorio para poder valorizar y preservar el patrimonio.

\section{Metodología}

\section{Área de estudio}

La Casona San Juanista se encuentra ubicada en la ciudad de Riobamba en el nuevo campus de la Universidad Nacional de Chimborazo. Caracterizada por ser una de las edificaciones más emblemáticas de la ciudad debido a que fue construida a principios del siglo XX, por lo que la fachada de este patrimonio arquitectónico difiere de las edificaciones que rodean cómo se puede observar en la Figura 1 a. Debido a su interés arquitectónico, parte de la Carrera de Arquitectura funcionan en esta edificación. La Iglesia de la "Compañía de Jesús" es una edificación de gran valor histórico y arquitectónico de la ciudad de Quito. Se encuentra ubicada en el Distrito Metropolitano de Quito dentro de su Centro Histórico. Este templo 
religioso es considerado una de las joyas arquitectónicas más importantes del continente americano y del mundo, principalmente por su espectacular fachada de estilo barroco levantada en piedra gris de origen volcánico como se ilustra en la Figura 1 b.

a)
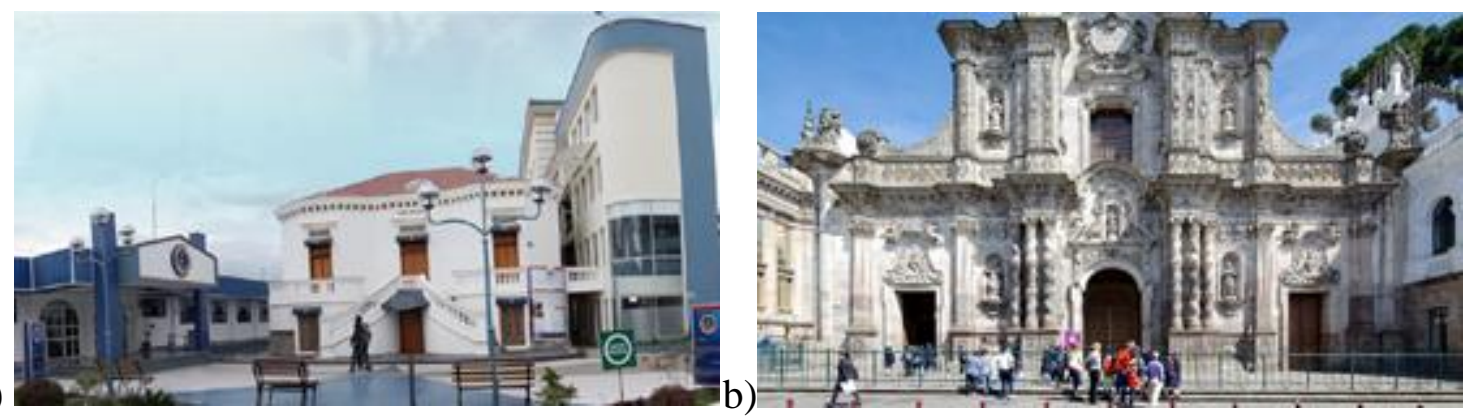

Figura 1.- imágenes de las fachadas registradas con el Escáner Láser Faro Focus 3D 130

Fuente: Trabajo de campo

Elaborado por: Los autores

\section{Instrumentación}

La tecnología de escaneo láser permite obtener modelos tridimensionales precisos de estructuras y componentes de instalaciones industriales; tiene la operatividad de una estación total con las ventajas de la toma de datos por un escáner láser (Owda et al. 2018). Por lo tanto, el escáner láser 3D que se utilizó para el presente proyecto es el de FARO 3D 130 ya que utiliza tecnología láser para generar modelos tridimensionales detalladas de geometrías y entornos complejos en minutos. El FARO Laser Scanner Focus3D 130 es un escáner láser terrestre (TLS) de medición por rebote y alta velocidad, este equipo es el método más eficiente de medición y documentación tridimensional. Se posiciona junto al teodolito y la estación total como herramienta indispensable para medir el entorno construido y las geometrías complejas de grandes estructuras de ingeniería.

Este equipo es un revolucionario escáner láser 3D de alto rendimiento para la medición y documentación detallada con una intuitiva pantalla táctil de sencillo uso. Cuatro veces más ligero y cinco veces más pequeño que su predecesor tiene la capacidad de registrar 976.000 puntos por segundo y posee cámara integrada de $70 \mathrm{Mpx}$ (Megapixeles) (Martínez et al. 2018). El escaneo láser utilizado tiene características de suma importancia como a) Precisión de distancias de hasta $\pm 2 \mathrm{~mm}$, medición desde $0,6 \mathrm{~m}$ a $130 \mathrm{~m}$; b) Velocidad de medición de hasta 976.000 puntos/s; c) Pantalla táctil intuitiva en cual las funciones de control y escaneado mediante la interfaz táctil proporcionan una facilidad de uso y control sin precedentes; d) Diseño ultra portátil que permite el funcionamiento sin dispositivos externos; 
e) es pequeño y compacto, pues posee unas dimensiones de $24 \times 20 \times 10 \mathrm{~cm}$ y un peso de 5,0 $\mathrm{kg}$; f) Tiene el control remoto WLAN cual permite iniciar, detener, ver o descargar escaneos de forma remota; g) Cámara a color integrada con superposición de color sin paralelaje de 70Mpx y automática; h) Batería integrada de iones de litio que proporciona hasta cinco horas de funcionamiento y puede cargarse durante el servicio.

Aunque la mayoría de los escáneres actuales pueden colectar datos girando $360^{\circ}$; esto no es necesario en muchos casos, por lo tanto, se necesita definir el área a escanear. El FARO Laser Scanner Focus3D 130 tiene actualmente un campo de visión horizontal/vertical de $360^{\circ} / 305^{\circ}$, teniendo un panel de control en el que se puede definir el área a escanear de forma rápida y aproximada (Martínez et al. 2018).

La resolución es otro punto a definir ya que ésta se encuentra determinada por el detalle de menor tamaño que se necesite reconocer en el producto final; por tanto, está directamente relacionada con la escala del producto final. En superficies uniformes como una pared se necesitan menos puntos para modelar este objeto, mientras tanto en superficies irregulares se necesitan más puntos, incluso a veces más de los que el escáner podría capturar (Oreni et al. 2014).

Mientras mayor sea la resolución del producto, se necesitará escanear más puntos y un mayor tiempo de captura de los datos; además que el tamaño de los datos almacenados aumentará (Buill et al. 2013; Fassi et al. 2013). La mayoría de los escáneres trabajan con un ángulo constante entre dos puntos consecutivos (basado en coordenadas polares), por lo cual la resolución del modelamiento estará definida para una cierta distancia del objeto a modelar hasta el escáner (Frolich et al. 2016; Gomes et al. 2014). Los puntos escaneados a una mayor distancia tendrán una menor resolución, mientras que los puntos más cercanos tendrán una más alta resolución (Koller et al. 2009).

La "English Heritage" es una institución de Reino Unido con gran experiencia en la Gestión de Entornos Históricos (Barber y Mills, 2007), la cual elaboró un estudio cuyo fin fue determinar la apropiada resolución para un escaneo, basado en ese planteamiento se puede determinar las propiedades para la toma de datos geoespaciales.

\section{Toma de datos y modelación 3D directo a partir de la nube de puntos}

Una vez determinados los parámetros para el levantamiento de datos, como se ilustra en la Tabla 1, se comienza con el proceso de escaneado, el cual es totalmente automático. Los puntos geoespaciales se almacenan en la memoria interna del escáner; al finalizar el proceso se genera una imagen 2D del área escaneada que se puede visualizar en pantalla. Según la resolución elegida y el área escaneada, el proceso puede durar desde cinco minutos hasta dos horas o más. 
Tabla 1. Parámetros para toma de datos con el escáner láser Faro Focus 3D 130

\begin{tabular}{lll}
\hline \multicolumn{1}{c}{ Parámetros } & Casona San Juanista & Iglesia Compañía de Jesús \\
\hline Perfil & Exterior hasta 20 metros & Exterior hasta 20 metros \\
Resolución & 01 -abr & 01 -abr \\
Número de escaneos & 11 escenas & 4 escenas frontales \\
Tiempo de escaneo & $10: 23$ & $31: 20: 00$ \\
& Vertical: -60 a 90 grados & Vertical: -60 a 90 grados \\
Ángulos & Horizontal: 0 a 360 grados & Horizontal: 0 a 360 grados \\
& Clinómetro, brújula, altíme- & Clinómetro, brújula, altímetro \\
Sensores activados & tro y GPS & y GPS \\
Color del escaneo & Activado & Activado \\
Tipo de medición ponderada & Ponderada al centro & Ponderada al centro \\
& Clear contour activado & Clear contour activado \\
Configuración avanzada & Clear sky activado & Clear sky activado \\
& Rango normal & Rango normal \\
\hline
\end{tabular}

Fuente: Trabajo de campo

Elaborado por: Los autores

Al conocer de antemano la forma de un objeto 3D se lo puede describir con primitivas geométricas detectadas automáticamente a partir de la nube de puntos; estas formas se ajustan a puntos determinados asumiendo el algoritmo que es una forma ideal. El producto final de un proceso de modelado 3D es una malla de la superficie del objeto, la cual conecta todos los puntos de la nube con pequeños triángulos.

El proceso se realiza mediante una interpolación de los puntos tridimensionales para crear una representación completa, por lo cual se establece una serie de pasos a desarrollarse: reducción del ruido y eliminación de errores groseros, muestreo, mallado, eliminación de vacíos y optimización de la malla. 


\section{Generación del Ambiente Virtual}

Un ambiente virtual es una interfaz que permite la interacción y visualización en tiempo real de objetos creados en un ordenador. Sin embargo, no sólo deben considerarse como partes primordiales de estos ambientes a los elementos propios de su interfaz (texto, gráficos, sonidos, animaciones, hipervínculos, entre otros), el objetivo fundamental radica en el alcance final obtenido al instaurarlo (Andrade y Narea, 2011; Maravelakis et al. 2013). Las características más importantes que se consideró para la generación de ambientes virtuales tanto de la Casona San Juanista como de la Iglesia Compañía de Jesús son:

- Expresión en un lenguaje gráfico tridimensional.

- Comportamiento dinámico y operación en tiempo real.

- Funcionamiento basado en la incorporación del usuario en el medio digital.

- Capacidad de reaccionar ante el usuario ofreciendo en su modo más complejo una experiencia inmersiva, interactiva y multisensorial.

Los modelos tridimensionales realizados en nuestro estudio fueron exportados a formatos específicos, con el fin de divulgar los datos generados en software de libre acceso disponible para el usuario como se ilustra en la Tabla 2.

Con respecto a la generación del ambiente virtual de la Casona San Juanista se realizó de los exteriores no solo de la fachada sino también de las edificaciones que rodean a la edificación puesto que uno de los parámetros era la toma de puntos hasta 20 metros desde cada escena. Tener un registro geométrico no solo de la fachada sino también de las edificaciones aledañas es importante para las aplicaciones arquitectónicas de este patrimonio cultural.

Para la generación del ambiente virtual de la Iglesia Compañía de Jesús se consideró los elementos frontales de la fachada ya que las escenas fueron cuatro frontales lo que permitió obtener el mayor registro geométrico de la fachada y los elementos que se encuentran frente a ella hasta 20 metros.

\section{Resultados}

La Casona San Juanista es característica por la complejidad que presenta en las molduras de su fachada. Por ello se realizó 11 escaneos cada uno con una resolución de 10240x4267 con medición ponderada al centro, la precisión de medición $\pm 20 \mathrm{~mm}$. La cantidad de puntos geoespaciales tomados fueron 156,223,503. La unificación de escaneos se realizó por coincidencia de planos cuya tensión media de referencia fue 0,002, lo cual indica que el enlace se realizó bajo los parámetros de tolerancia. Para evitar perder detalles de las molduras se optó por el mallado texturizado en el plano X, en referencia a la estación del escáner para después tener un mallado compuesto unificando los mallados antes mencionados como se ilustra en la Figura 2. 


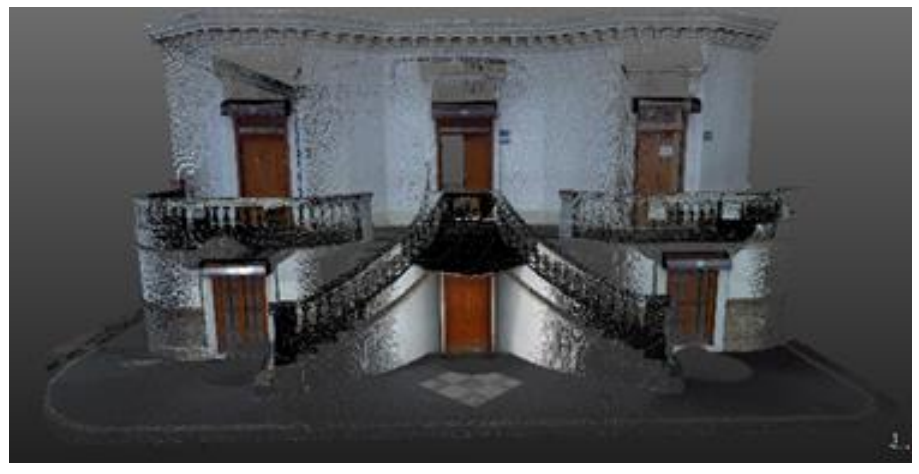

Figura 2.- Ambiente virtual 3D de la fachada de La Casona

Fuente: Trabajo de gabinete

Elaborado por: Los autores

Con los once levantamientos se obtuvo puntos geoespaciales de La Casona y sus alrededores como se visualiza en la Figura 3, lo que permite realizar recorrido de los exteriores de La Casona. Con este tipo de información geoespacial es posible realizar recorridos virtuales utilizando el software Trimble Real Works, mismo que permite visualizar la edificación arquitectónica desde diferentes ángulos sin perder la visualización de los detalles de las molduras.

Debido a que la edificación fue construida a inicios del siglo XX, requiere restauraciones para lo cual son de gran utilidad los registros geométricos no solo de la fachada de la edificación sino también de las edificaciones aledañas a la misma. Por ello se obtuvo el registro geométrico y un video del recorrido de los exteriores de la Casona San Juanista.

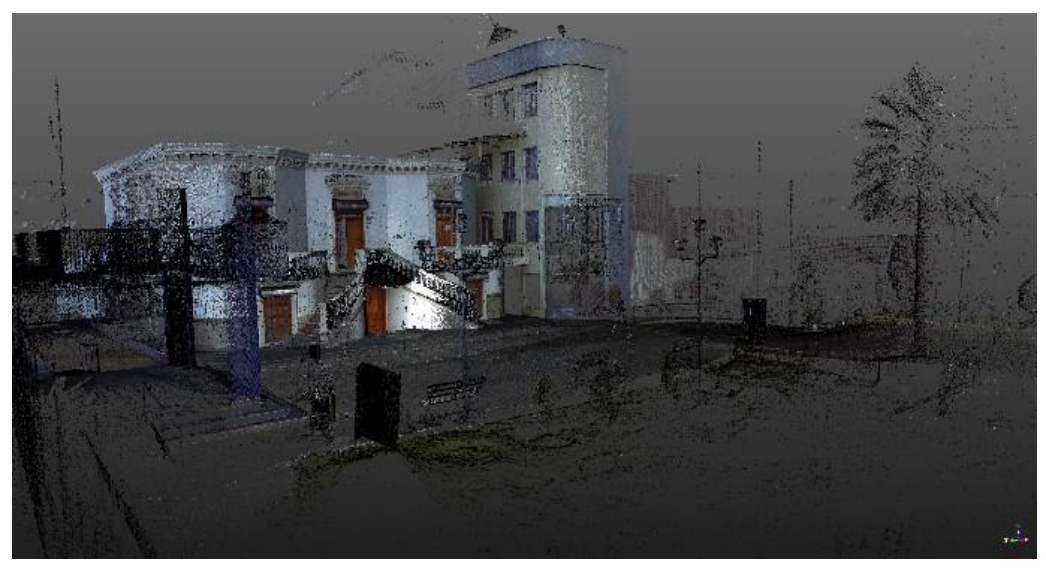

Figura 3.- Visualización de los exteriores de La Casona

Fuente: Trabajo de gabinete

Elaborado por: Los autores 
La Fachada de la Iglesia "La Compañía de Jesús" por su estructura, molduras y falta de accesibilidad a la parte superior, se realizó cuatro escaneos frontales con una precisión de medición de $\pm 25 \mathrm{~mm}$. La cantidad de puntos obtenidos fue de $108,160,946$. Una vez obtenidos los puntos se realizó la unificación de los escaneos de forma automática debido a que la posición de las estaciones fue frontal. El enlace de los escaneos presentó un valor medio de la tensión de referencia de 0,003, indicando así que el enlace se realizó correctamente. Debido a las molduras que posee este Patrimonio Cultural, se optó por utilizar el mallado texturizado para obtener el modelo tridimensional como se ilustra en la Figura 4. En la parte superior existen unos gaps debido al ángulo de toma de puntos por cada una de las escenas de escaneo, por lo que se procuró realizar una planificación de toma de datos que cubra la mayor área posible debido a que no se podía acceder a la parte superior de la fachada por protocolos de seguridad que mantiene la administración de la Iglesia Compañía de Jesús

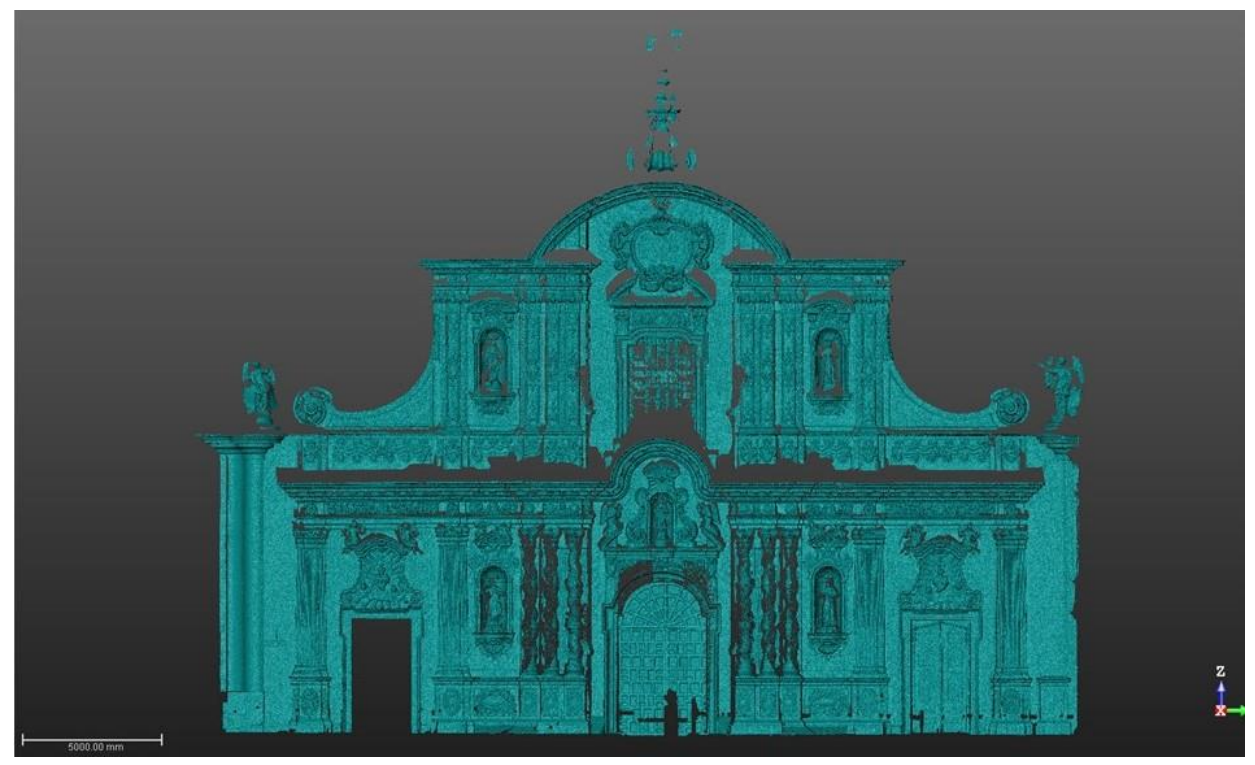

Figura 4.- Modelo 3D del Fachada de la Iglesia "La Compañía de Jesús"

Fuente: Trabajo de gabinete

Elaborado por: Los autores

Aun cuando se realizó una depuración de los puntos obtenidos en cada una de las escenas de escaneo, la cantidad de puntos utilizados para la realización de las mallas a través de triangulaciones aumentó considerablemente el tamaño del archivo, lo cual limitó la exportación a tres formatos (RWP, DXF, AVI). No se logró exportar la malla a formato DXF debido a que supera los 32.000 vértices especificado para este formato; por ende, no se obtuvo la malla en formato VRML, ya que este formato depende del DXF. En el caso del formato PDF 3D se presentó dificultades al exportar la malla, pues actualmente los Plugins no exportan este tipo de modelos tridimensionales. En la Tabla 2 se resume la exportación de los modelos a formatos RWP, DXF, KMZ, VRML, PDF 3D. 
Tabla 2. Resumen de la exportación de los modelos a diversos formatos

\section{Formato \\ Fachada de la Iglesia "La Compañía de Jesús" y Fachada de "La Casona San Juanista"}

RWP Trimble RealWorks exportó el modelo tridimensional de mallas en este formato.

DXF Trimble RealWorks no logró exportar la malla debido a que supera la capacidad de 32.000 vértices especificado para este formato.

Trimble RealWorks intentó exportar la malla en este formato, pero se presentó

KMZ dificultad debido al tamaño del archivo (número de vértices) saturando al equipo.

Para exportar a este formato se requiere previamente el formato .dxf, el cual no

VRML se obtuvo por la excesiva cantidad de vértices que posee el modelo tridimensional.

PDF 3D Este formato presenta dificultades al exportar mallas, actualmente los diferentes Plugins no exportan mallas.

AVI

El programa Trimble RealWorks permitió realizar un video del modelo tridimensional en este formato.

Fuente: Trabajo de gabinete

Elaborado por: Los autores

La obtención de los modelos 3D de los monumentos de Patrimonio Cultural antes mencionados requieren metadatos 3D unificados como lo propone Maravelakis et al. (2013), para un mejor almacenamiento y manejo de datos geoespaciales considerando que se requieren de millones de puntos para que los modelos tridimensionales tengan un alto nivel de precisión como los que se obtuvieron en esta investigación. Por otro lado, para la captura y almacenamiento de datos se llevó a cabo protocolos de acuerdo con la estructura de los monumentos lo cuales son similares a los propuestos por Buills et al. (2013). Cabe mencionar que otros proyectos se basan en esta tecnología para la digitalización de monumentos patrimoniales implementando más tecnologías como la fotogrametría como es el caso de Martínez et al. (2018); sin embargo, la tecnología vanguardista es el escáner láser (Bernat, 2014; Owda, 2018) con la cual se ha obtenido resultados similares, respecto a resolución, rango de error y calidad del producto final, a los del presente proyecto. 
El presente proyecto al igual que los proyectos realizados por Fassi et al. (2013) y Koller (2009), presentó dificultades al momento de exportar los modelos a diferentes archivos como se ilustra en la Tabla 1, situación que ha venido siendo un reto debido al manejo de la cantidad de datos tomados para este tipo de monumentos. De esta forma, como manifiesta Peres et al. (2015) es esperado que, a partir de iniciativas de esta naturaleza, sean mejor direccionadas a la concepción de proyectos de preservación y valoración de las edificaciones de interés histórico, confiriendo unidad al ambiente urbano de modo a promover la efectiva preservación, educación e integración de la comunidad a su patrimonio histórico-cultural.

\section{Conclusiones.}

- Las tensiones entre todos los pares de referencia sirven para ubicar a los escaneos, donde el valor medio de la tensión de referencia indica si los escaneos están correctamente enlazados. El enlace de los escaneos de La Casona tiene 0,002 de valor medio de la tensión de referencia, mientras en el enlace de los escaneos de la Fachada de la Iglesia "La Compañía de Jesús" tiene 0,003; por lo tanto, el valor medio de la tensión de referencia de los escaneos es óptimo en las dos áreas de trabajo.

- Para realizar el escaneo de cada área de trabajo se tomó diferente número de escaneos, esto depende de la complejidad de la estructura y principalmente sus facilidades de acceso para realizar el levantamiento de la información. Cabe mencionar que al generar los modelos tridimensionales de las áreas de estudio se logró determinar que cada una de ella tiene un proceso diferente, que varía en función de la forma de su estructura, nivel de detalle, cantidad de escaneos realizados y número de puntos; cabe tomar en cuenta que la aplicación dada al modelo influye en el momento de generarlo.

- El proceso de exportación de los modelos tridimensionales varió de acuerdo con las ventajas y desventajas que presentó cada formato. El modelo tridimensional de La Casona se logró exportar a RWP DXF, VRML, AVI, mientras el modelo tridimensional de la Fachada de la Iglesia "La Compañía de Jesús" fue el más complicado de exportar pues su cantidad de vértices limita la exportación a diversos formatos y que estos archivos sean visualizados sin dificultad, por ello se logró exportar al formato RWP, DXF y AVI.

- El campo de aplicación dentro del desarrollo local de estas tecnologías se basa en la construcción de vínculos entre la población local y su desarrollo, el territorio y lo espacial o aéreo. Adicional, se puede generar política pública para preservar, valorar y manejar el patrimonio cultural inventariado, actualizando información y perfeccionándola, visualizando a manera de un todo o detallado acorde a la necesidad que permite el entendimiento de la dinámica del entorno.

\section{Referencias bibliográficas.}

1. Andrade, L., y Narea, P. (2011). Análisis, diseño e implementación de un ambiente virtual 3D y objetos de información para la Regional Austro del Ministerio de Turismo. Universidad Politécnica Salesiana, Quito.

2. Apollonio, F. I., Gaiani, M., y Benedetti, B. (2012). 3D reality-based artefact models for the management of archaeological sites using 3D Gis: a framework 
starting from the case study of the Pompeii Archaeological area. Journal of archaeological Science, 39(5), 1271-1287.

3. Barber, D., y Mills, J. (Eds.). (2007). 3D laser scanning for heritage: Advice and guidance to users on laser scanning in archaeology and architecture. English Heritage.

4. Bermúdez, D. P., de la Llave, R. C., Cabrera, J. S., y Tubío, F. D. P. M. (2016). Metodología para la reconstrucción virtual interactiva en modo videojuego del patrimonio cultural. Aplicación al castillo medieval de Torreparedones (Baena). EGA Expresión Gráfica Arquitectónica, 21(28), 278-287.

5. Bernat, M., Janowski, A., Rzepa, S., Sobieraj, A., y Szulwic, J. (2014). Studies on the use of terrestrial laser scanning in the maintenance of buildings belonging to the cultural heritage. 14th Geoconference on Informatics, Geoinformatics and Remote Sensing, SGEM. ORG, Albena, Bulgaria, 3, 307-318.

6. Buill, F., Andrés, M. A. N., y Marimón, J. R. (2013). Técnicas de captura masiva de datos para el levantamiento y modelado de monumentos medievales. EGA Expresión Gráfica Arquitectónica, 18(21), 62-73.

7. Echeverría, E., Castaño, E., Casa, F. D., y Chías, P. (2017). Documental studio and 3D recreation of the San Ildefonso's School façade. Universidad de Alcalá, Alcala de Henares.

8. Fassi, F., Fregonese, L., Ackermann, S., y De Troia, V. (Febrero, 2013). Comparison between laser scanning and automated $3 \mathrm{~d}$ modelling techniques to reconstruct complex and extensive cultural heritage areas. International Archives of the Photogrammetry, Remote Sensing and Spatial Information Sciences. 3D Virtual Reconstruction and Visualization of Complex Architectures, Turin-Italy.

9. Frohlich, R., Kato, Z., Tremeau, A., Tamas, L., Shabo, S., y Waksman, Y. (Diciembre, 2016). Region based fusion of 3D and 2D visual data for Cultural Heritage objects. 23rd International Conference on Pattern Recognition. IEEE, Cancun-México, 2404-2409.

10. Gomes, L., Bellon, O. R. P., y Silva, L. (2014). 3D reconstruction methods for digital preservation of cultural heritage: A survey. Pattern Recognition Letters, $50,3-14$.

11. Guidi, G., Micoli, L. L., Gonizzi, S., Brennan, M., y Frischer, B. (2015). Imagebased 3D capture of cultural heritage artifacts an experimental study about 3D data quality. Digital Heritage, 2, 321-324.

12. Koller, D., Frischer, B., y Humphreys, G. (2009). Research challenges for digital archives of 3D cultural heritage models. Journal on Computing and Cultural Heritage (JOCCH), 2(3), 7.

13. Manso Alconada, M. I. (2017). Estudio acústico virtual de la cripta del Hospital Tavera (Toledo): implementación de tecnologías escáner láser 3D y análisis espacial del modelo. Universidad Politécnica de Madrid, Madrid-España.

14. Mañana, P., Paz, A., y Blanco, R. (2008). Una experiencia en la aplicación del Láser Escáner 3D a los procesos de documentación y análisis del Patrimonio Construido: su aplicación a Santa Eulalia de Bóveda (Lugo) y San Fiz de Solovio (Santiago de Compostela). Arqueología de la Arquitectura, (5), 15-32.

15. Maravelakis, E., Konstantaras, A., Kritsotaki, A., Angelakis, D., y Xinogalos, M. (Julio, 2013). Analysing user needs for a unified 3D metadata recording and 
exploitation of cultural heritage monuments system. International Symposium on Visual Computing. Springer, Berlin, Heidelberg, 138-147.

16. Martínez Rubio, J., Fernández Martín, J. J., y San José Alonso, J. I. (Marzo, 2018). Implementación de escáner $3 \mathrm{~d}$ y fotogrametría digital para la documentación de la iglesia de La Merced de Panamá. Revista de Expresión Gráfica Arquitectónica ,23 (32), 208-219.

17. Oreni, D., Brumana, R., Della Torre, S., Banfi, F., y Previtali, M. (2014). Survey turned into HBIM: the restoration and the work involved concerning the Basilica di Collemaggio after the earthquake (L'Aquila). ISPRS Annals of the Photogrammetry, Remote Sensing and Spatial Information Sciences, 2(5), 267.

18. Owda, A., Balsa, J., y Fritsch, D. (2018). Methodology for digital preservation of the cultural and patrimonial heritage: generation of a 3D model of the Church St. Peter and Paul (Calw, Germany) by using Laser scanning and digital photogrammetry. Sensor Review, 38 (2), 282-288.

19. Peres, O., Polidor, M., y Tomiello, F. (2015). Patrimonio Cultural y SIG: escalas de visualización y preservación. Ciencias Espaciales, 8 (2), 41-28.

20. Piedecausa, B., Mateo, J., y Pérez, J. (2015). Enseñanza de sistemas BIM en el ámbito universitario. Congreso Internacional BIM/ Encuentro de Usuarios BIM. Universidad Politécnica de Valencia, Valencia, España, 93-101.

21. Redondo, E., y Adas, A. (2014). Una nueva metodología para la documentación y modelado $3 \mathrm{~d}$ de elementos del patrimonio arquitectónico: Un estudio de viabilidad y de caso. Los miradores colgantes de madera "Roshan" en la ciudad histórica de Jeddah. EGA Expresión Gráfica Arquitectónica, 19(24), 176-187.

22. Santana, M., y Lerma, J. (2008). Teoría y práctica del Escaneado Láser Terrestre. Material de aprendizaje en aplicaciones prácticas. Herramientas de aprendizaje para el levantamiento tridimensional avanzado en la conciencia de riesgos. 3D Risk Mapping-Agencia Flamenca del programa europeo Leonardo Da Vinci, 241.

23. Siabato, W. (2018). Sobre la evolución de la información geográfica: las bodas de oro de los SIG. Cuadernos de Geografía: Revista Colombiana de Geografía, 27(1), 1-9.

24. Tamayo, C. V., y Leite, E. (2015). Las tecnologías de la información y las comunicaciones como herramientas para la gestión del patrimonio cultural con una visión emprendedora. HOLOS, 8, 290-303.

25. Xu, Z., Wu, L., Shen, Y., Li, F., Wang, Q., y Wang, R. (2014). Tridimensional reconstruction applied to cultural heritage with the use of camera-equipped UAV and terrestrial laser scanner. Remote Sensing, 6(11), 10413-10434.

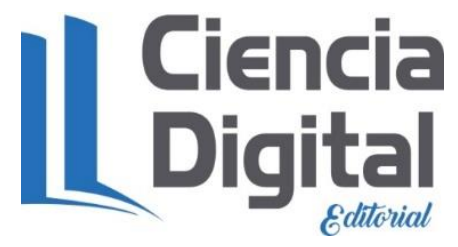




\section{PARA CITAR EL ARTÍCULO INDEXADO.}

Velastegui Cáceres, L., Velastegui Cáceres, J., Noboa Silva, V., \& Moyon Gusqui, M. (2019). Obtención de modelos 3D de estructuras de patrimonio cultural-urbano con el uso de tecnología escáner láser y SIG, como instrumentos para el desarrollo local. Ciencia Digital, 3(4.1), 51-65. https://doi.org/10.33262/cienciadigital.v3i4.1.980

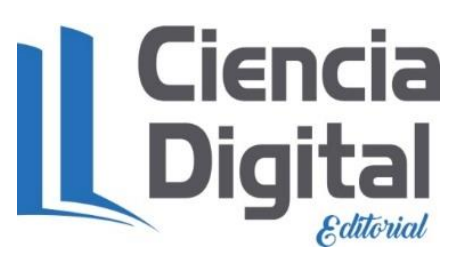

El artículo que se publica es de exclusiva responsabilidad de los autores y no necesariamente reflejan el pensamiento de la Revista Ciencia Digital.

El artículo queda en propiedad de la revista y, por tanto, su publicación parcial y/o total en otro medio tiene que ser autorizado por el director de la Revista Ciencia Digital.
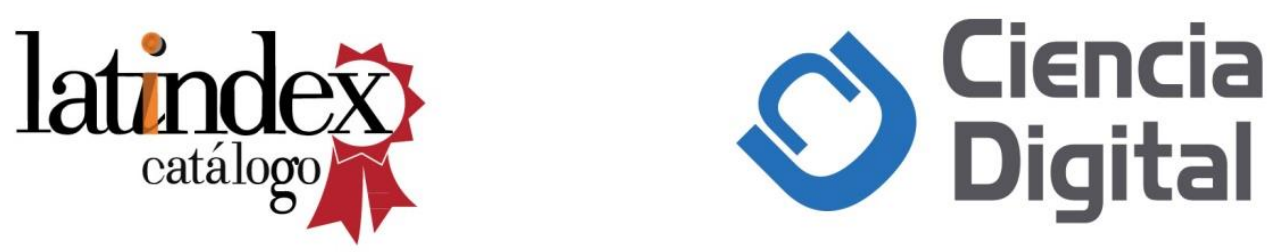